\title{
Unilateral Multifocal Type 2 Papillary Renal Cell Carcinoma in Adolescence. A Case Report
}

\author{
Sol Yoon ${ }^{1}$, Jae Hwi Choi ${ }^{1}$, Sin Woo Lee ${ }^{1}$, Seong Uk Jeh", Jeong Seok Hwa', \\ Jae Seog Hyun ${ }^{1}$, Ky Hyun Chung ${ }^{1}$, Hyun Oh Park ${ }^{2}$, Jong Sil Lee ${ }^{3}$, See Min Choi ${ }^{1}$ \\ ${ }^{1}$ Department of Urology, Gyeongsang National University Hospital, Institute of Health Science, Gyeongsang \\ National University, Jinju, Korea \\ ${ }^{2}$ Department of Thoracic and Cardiovascular Surgery, Gyeongsang National University Hospital, \\ Gyeongsang National University School of Medicine, Jinju, Korea \\ ${ }^{3}$ Department of Pathology, Gyeongsang National University Hospital, Gyeongsang National University \\ School of Medicine, Jinju, Korea
}

\begin{abstract}
Papillary renal cell carcinomas (RCCs) can be classified into 2 subtypes (types 1 and 2), depending on their characteristic cytogenetics, immunostaining profiles, and gene-expression profiles. Compared with type 1 papillary RCCs, type 2 papillary RCCs are relatively rare and show more aggressive features. For those reasons, they are associated with a worse prognosis. A 13-year-old patient was admitted to the hospital with right kidney mass. A laparoscopic radical nephrectomy was performed, and results of the histopathologic examination confirmed it to be type 2 papillary RCC. Type 2 papillary RCCs are rarely found in unilateral and multifocal forms, especially in adolescence. Here, we report the unique case of papillary RCC at a young age. (Korean J Urol Oncol 2019;17: 70-73)
\end{abstract}

Key Words: Type 2 papillary renal cell carcinoma - Unilateral $\cdot$ Multifocal $\cdot$ Adolescence

Among the types of renal cell carcinomas (RCCs), papillary RCCs are the second most common type and are classified into 2 subtypes (types 1 and 2), depending on their characteristic cytogenetics, immunostaining profiles and gene-expression profiles. ${ }^{1}$ Compared to type 1 papillary RCCs, type 2 papillary RCCs are relatively rare and show more aggressive features. ${ }^{2}$ As with other types of RCCs, most cases are asymptomatic and found incidentally through imaging study. Treatment involves partial or radical nephrectomy, and is followed by a histological test to confirm the diagnosis. The authors of this study report

Received January 8, 2019, Revised February 18, 2019,

Accepted February 27, 2019

Corresponding Author: See Min Choi

Department of Urology, Gyeongsang National University Hospital,

79 Gangnam-ro, Jinju 52727, Korea

E-mail: choi9854@hanmail.net

Tel: +82-55-750-9589, Fax: +82-55-750-9589

ORCID code: https://orcid.org/0000-0002-6599-2640 on a case of unilateral and multifocal papillary RCC occurring in adolescence.

\section{CASE REPORT}

\section{Review of clinical data}

The clinical profile of the patient was obtained from the electronic medical records of our institution. We obtained approval for the research from the Gyeongsang National University Hospital Institutional Review Board (approval number: 2019-02-001).

\section{Clinical findings}

A 13-year-old patient without any medical or surgical history was admitted to the hospital with a mass in the right kidney. The patient did not have a family history of kidney cancer and did not show any specific findings on physical examination. 
The patient was found to have normal complete blood count, blood chemistry tests, urine analysis, and urine culture. Chest $\mathrm{X}$-ray showed no specific findings. Computed tomography scan showed a cystic and solid tumor of approximately $7.5 \mathrm{~cm}$ in the right kidney. Neither perirenal fat infiltration, lymph node invasion nor distant metastasis were seen (Fig. 1). The patient underwent a right transperitoneal laparoscopic radical nephrectomy.

\section{Pathological findings}

In gross pathological findings, a kidney with a size of 12 $\mathrm{cm} \times 8 \mathrm{~cm} \times 3.5 \mathrm{~cm}$ was incised, and a nodule with a yellow cystic change of $3.5 \mathrm{~cm}$ that protruded slightly was identified. In addition, a number of cystic lesions were identified (Fig. 2). The final histological report identified papillary RCCs at 3 sites, with sizes of $3.5,3.5$, and $1.5 \mathrm{~cm}$. The patient was found to have Fuhrman grade I and a clear cell component of less than $10 \%$. The renal mass was confined to the inside of the

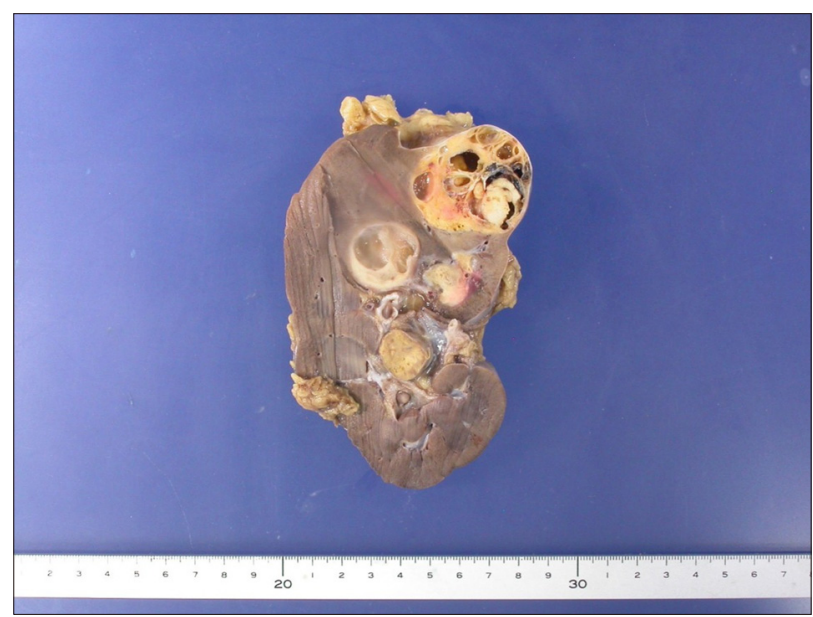

Fig. 2. Gross feature of resected right kidney. The kidney shows multiple tumors. The largest one is composed of solid and cystic areas.
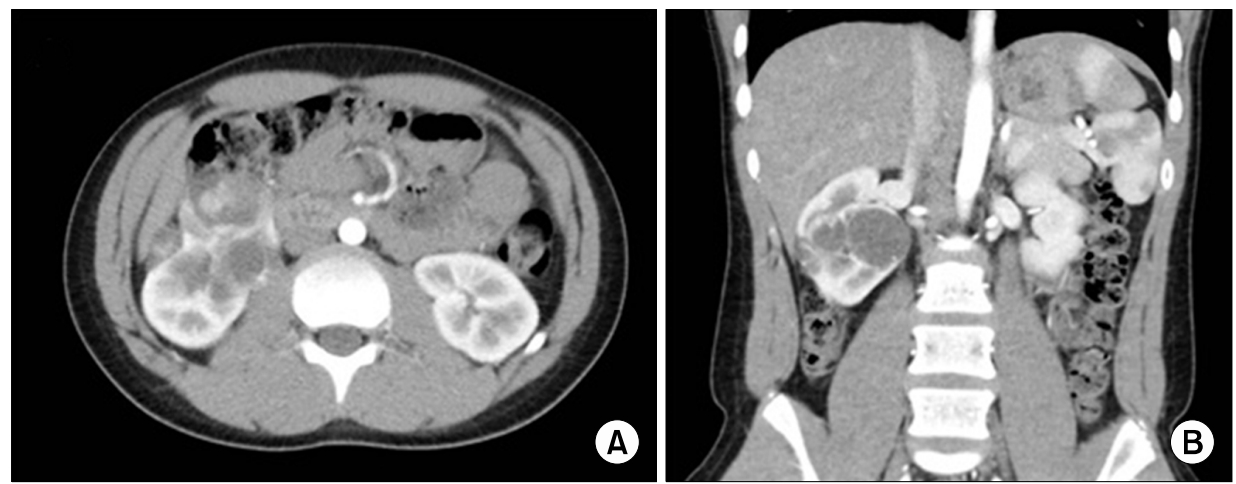

Fig. 1. Contrast-enhanced abdominal computed tomography showing $7.5-\mathrm{cm}$ size multiple cystic and solid tumor in right kidney. Transverse section (A) and coronary section (B).
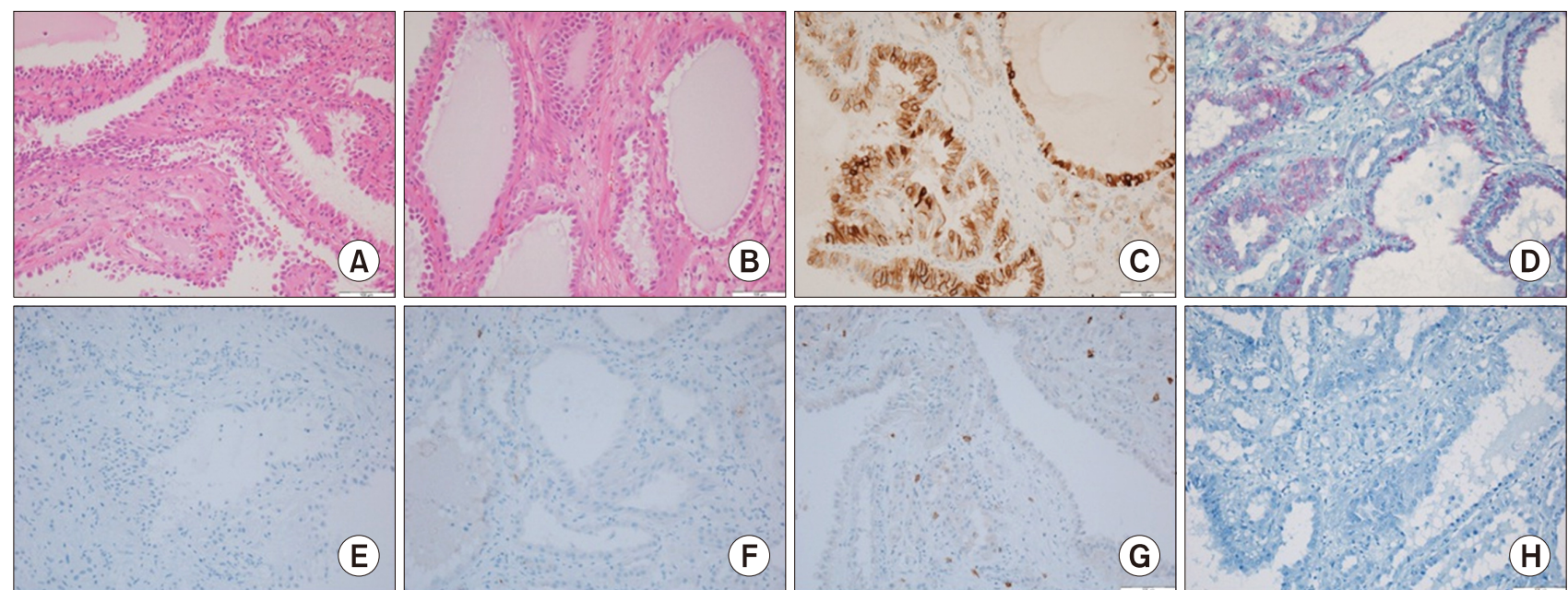

Fig. 3. Histologic and immunohistochemical findings of tumor. (A, B) The tumor is composed of papillary proliferation of oncocytes with eosinophilic finely granular cytoplasm and shows nuclear pseudostratification (hematoxylin and eosin). The tumor is positive for EMA (C), AMACR (D); and negative for CD10 (E), CK7 (F), CD117 (G), and HMB45 (H) (original magnifications: A-H, $\times 200$ ). 
kidney, and there was no infiltration into renal veins and the circumferential resection margin was negative. Immunohistochemical staining on each renal mass, yielded positive results for epithelial membrane antigen (EMA) and Pax8 stain, but negative staining for CD10, cytokeratin (CK) 7, and CD117. The masses reacted non-specifically for alpha methylacyl-CoAracemase (AMACR) staining (Fig. 3). The results confirmed the diagnosis of multiple type 2 papillary RCCs, and T stage pT1a (m).

\section{Hospital course}

Follow-up imaging at 7 months showed no signs of recurrence. The patient's progress is currently being monitored on an-outpatient basis.

\section{DISCUSSION}

The subtypes of papillary RCCs have their own characteristic gross and histological features. Papillary RCCs can be classified into two subtypes, depending on their cytological features. Type 1 is composed of basophilic cells with a low nuclear grade; its neoplastic cells are made up of single layers, and foamy macrophages and psammoma bodies can be easily observed. Type 2 is composed of eosinophilic cells with a high nuclear grade, in the form of pseudostratified or multilayered large cells. Because these 2 types have different prognoses, it is meaningful to distinguish them. ${ }^{3}$

In this case, tumor histology showed papillary proliferation with eosinophilic finely granular cytoplasm and nuclear pseudostratification. The Fuhrman grade classifies RCC into grade 1 to 4 , according to their nuclear sizes, shapes, and presence of prominent nucleoli. Originally, it was applied as a prognostic tool specifically for clear cell RCC, but a study by Klatte et al. ${ }^{4}$ reported that it could function as the standard grading system for papillary RCCs, as well. Therefore, Fuhrman grade 1 papillary RCCs may be associated with a better prognosis.

Specific characteristics of papillary RCCs can be confirmed through immunohistochemistry. Most papillary RCCs are positive for CK7, vimentin, AMACR, Pax2, and Pax8, but negative for CD117 and carbonic anhydrase-IX (CA-IX). The immunohistochemical findings in this case showed positive reactions for EMA, Pax8, and AMACR, but negative reactions for CD117, CK7 and CD10. Considering that CK7 is usually expressed in papillary RCCs, with some exceptions, the im- munochemistry results of this case are generally consistent with the known characteristics of papillary RCCs. ${ }^{5}$

Cytogenetic and molecular studies can also be used to distinguish papillary RCC subtypes. Papillary RCCs are characterized by trisomy in chromosomes 7 and 17, or by the loss of chromosome $\mathrm{Y}^{6}$ These characteristics are more commonly observed in type 1 , than in type 2 RCCs. In addition, MET gene mutation is a trait appearing uniquely in type 1.7

According to a study by Steffens et al., ${ }^{8}$ the 5-year cancerspecific survival rates of papillary RCCs and clear cell RCCs were estimated at $85.1 \%$ and $76.9 \%$, respectively, and papillary RCCs showed as better prognosis than clear cell RCCs. Pignot et al. ${ }^{3}$ reported that the overall survival rates of types 1 and 2 papillary RCCs were $89 \%$ and $55 \%$, respectively, and that type 2 had a worse prognosis than type 1 . On the other hand, there are several claims that the papillary RCC subtype does not affect the oncologic outcome. ${ }^{9}$ Therefore, additional research and discussion is needed to conclude this controversy.

Like clear cell RCCs, the follow-up plan is similar in papillary RCCs. A low risk patient who has undergone radical nephrectomy is recommended to perform initial postoperative image, physical examination and laboratory test within 3 to 12 months after surgery. If initial postoperative follow-up findings are normal, periodic follow-up tests are recommended for 5 years after surgery.

If recurrence occurs during follow-up in papillary RCCs, systemic therapy such as sunitinib, cabozantinib, and everolimus can be considered. And metastatectomy, stereotactic body radiation therapy and ablative therapy are also possible therapies. Several clinical trials are in progress in this regard. ${ }^{10}$

In summary, papillary RCC is a kidney cancer that is less common than clear cell RCC. Given the nature of kidney cancers, preoperative diagnosis can be difficult, but they can be confirmed by histological tests. Papillary RCCs are divided into 2 subtypes, depending on the characteristic gross and histological features, and type 2 has a poorer prognosis than type 1. Type 2 papillary RCC usually occurs in men between 60 and 70 years of age, but this case was confirmed as a unilateral multifocal kidney lesion, occuring in an adolescent male patient. This study reports the first case of an extremely rare type 2 papillary RCC. 


\section{CONFLICT OF INTEREST}

The authors claim no conflicts of interest.

\section{REFERENCES}

1. Sukov WR, Lohse CM, Leibovich BC, Thompson RH, Cheville JC. Clinical and pathological features associated with prognosis in patients with papillary renal cell carcinoma. J Urol 2012;187:54-9.

2. Reuter VE, Presti JC Jr. Contemporary approach to the classification of renal epithelial tumors. Semin Oncol 2000;27:12437.

3. Pignot G, Elie C, Conquy S, Vieillefond A, Flam T, Zerbib M, et al. Survival analysis of 130 patients with papillary renal cell carcinoma: prognostic utility of type 1 and type 2 subclassification. Urology 2007;69:230-5.

4. Klatte T, Anterasian C, Said JW, de Martino M, Kabbinavar FF, Belldegrun AS, et al. Fuhrman grade provides higher prognostic accuracy than nucleolar grade for papillary renal cell carcinoma. J Urol 2010;183:2143-7.

5. Skinnider BF, Folpe AL, Hennigar RA, Lim SD, Cohen C, Tamboli $\mathrm{P}$, et al. Distribution of cytokeratins and vimentin in adult renal neoplasms and normal renal tissue: potential utility of a cytokeratin antibody panel in the differential diagnosis of renal tumors. Am J Surg Pathol 2005;29:747-54.

6. Lager DJ, Huston BJ, Timmerman TG, Bonsib SM. Papillary renal tumors. Morphologic, cytochemical, and genotypic features. Cancer 1995;76:669-73.

7. Schmidt L, Duh FM, Chen F, Kishida T, Glenn G, Choyke $\mathrm{P}$, et al. Germline and somatic mutations in the tyrosine kinase domain of the MET proto-oncogene in papillary renal carcinomas. Nat Genet 1997;16:68-73.

8. Steffens S, Janssen M, Roos FC, Becker F, Schumacher S, Seidel $\mathrm{C}$, et al. Incidence and long-term prognosis of papillary compared to clear cell renal cell carcinoma: a multicentre study. Eur J Cancer 2012;48:2347-52.

9. Ha YS, Chung JW, Choi SH, Lee JN, Kim HT, Kim TH, et al. Clinical significance of subclassification of papillary renal cell carcinoma: comparison of clinicopathologic parameters and oncologic outcomes between papillary histologic subtypes 1 and 2 using the Korean Renal Cell Carcinoma Database. Clin Genitourin Cancer 2017;15:e181-6.

10. Armstrong AJ, Halabi S, Eisen T, Broderick S, Stadler WM, Jones RJ, et al. Everolimus versus sunitinib for patients with metastatic non-clear cell renal cell carcinoma (ASPEN): a multicentre, open-label, randomised phase 2 trial. Lancet Oncol 2016;17:378-88. 